\title{
Using Machine Learning to Partition Streaming Programs
}

\author{
ZHENG WANG and MICHAEL F. P. O’BOYLE, University of Edinburgh
}

Stream-based parallel languages are a popular way to express parallelism in modern applications. The efficient mapping of streaming parallelism to today's multicore systems is, however, highly dependent on the program and underlying architecture. We address this by developing a portable and automatic compilerbased approach to partitioning streaming programs using machine learning. Our technique predicts the ideal partition structure for a given streaming application using prior knowledge learned offline. Using the predictor we rapidly search the program space (without executing any code) to generate and select a good partition. We applied this technique to standard StreamIt applications and compared against existing approaches. On a 4-core platform, our approach achieves $60 \%$ of the best performance found by iteratively compiling and executing over 3000 different partitions per program. We obtain, on average, a $1.90 \times$ speedup over the already tuned partitioning scheme of the StreamIt compiler. When compared against a state-ofthe-art analytical, model-based approach, we achieve, on average, a $1.77 \times$ performance improvement. By porting our approach to an 8 -core platform, we are able to obtain $1.8 \times$ improvement over the StreamIt default scheme, demonstrating the portability of our approach.

Categories and Subject Descriptors: D.3.4 [Programming Languages]: Processors—Compilers, optimization; D.1.3 [Programming Techniques]: Concurrent Programming-Parallel programming

General Terms: Experimentation, Languages, Measurement, Performance

Additional Key Words and Phrases: Compiler optimization, multicore, machine learning, partitioning streaming parallelism

\section{ACM Reference Format:}

Wang, Z. and O’Boyle, M. F. P. 2013. Using machine learning to partition streaming programs. ACM Trans. Architec. Code Optim. 10, 3, Article 20 (September 2013), 25 pages.

DOI: http://dx.doi.org/10.1145/2512436

\section{INTRODUCTION}

Multicore processors are now mainstream, offering the promise of energy-efficient hardware performance [Asanovic et al. 2009]. To make use of such potential, however, new and existing applications must be written or transformed so that they can be executed in parallel. While there has been considerable research effort focused on (semi-) automatically transforming existing sequential programs into a parallel form [Tournavitis et al. 2009], it remains uncertain whether this is a long-term viable approach to achieving scalable parallelism [Asanovic et al. 2009].

An alternative approach has been to develop new high-level programming languages and models where the parallelism is explicit. Although the application developer

Z. Wang is currently affiliated with Lancaster University, UK.

A preliminary version of this article entitled "Partitioning Streaming Parallelism for Multi-cores: A Machine Learning Based Approach" by Z. Wang and M. O'Boyle appeared in the $19^{\text {th }}$ Internaional Conference on Parallel Architectures and Compilation Techniques (PACT) in 2010 [Wang and O’Boyle 2010].

Authors' addresses: Z. Wang (corresponding author) and M. F. P. O'Boyle, School of Informatics, University of Edinburgh, Edinburgh EH8 9AB, UK; email: zh.wang@ed.ac.uk.

Permission to make digital or hard copies of part or all of this work for personal or classroom use is granted without fee provided that copies are not made or distributed for profit or commercial advantage and that copies show this notice on the first page or initial screen of a display along with the full citation. Copyrights for components of this work owned by others than ACM must be honored. Abstracting with credit is permitted. To copy otherwise, to republish, to post on servers, to redistribute to lists, or to use any component of this work in other works requires prior specific permission and/or a fee. Permissions may be requested from Publications Dept., ACM, Inc., 2 Penn Plaza, Suite 701, New York, NY 10121-0701 USA, fax +1 (212) 869-0481, or permissions@acm.org.

(c) 2013 ACM 1544-3566/2013/09-ART20 $\$ 15.00$

DOI: http://dx.doi.org/10.1145/2512436 
has to think explicitly about parallel structure in the application, she is freed from implementation concerns. As an application may be ported to new platforms many times in its lifetime, this is a significant advantage.

One popular language domain focuses on streaming applications exposing task, data, and pipeline parallelism [Stephens 1997]. Parallelism in streaming languages is explicit and it is now the system's responsibility to effectively map this parallelism to the underlying hardware. Mapping is typically broken into two stages: partitioning the program graph into regions which are allocated to threads and then scheduling, which allocates the threads to the underlying hardware. This is by no means a new challenge and there is an extensive body of work on mapping task, data, and pipeline parallelism to parallel architectures using runtime scheduling [Ramamritham and Stankovic 1984], static partitioning [Kwok and Ahmad 1999; Sarkar 1991], analytical models [Culler et al. 1996; Navarro et al. 2009], heuristic-based mappings [Gordon et al. 2006], or ILP solvers [Kudlur and Mahlke 2008; Udupa et al. 2009]. They can each achieve good performance but are restricted in their applicability. Fundamentally, such approaches are based on the developers' view about the most significant costs of the target platform and typical programs, encoding a hardwired human-derived heuristic. However, as we show later, the best form of the program varies across programs and platforms.

The problem with hardwired heuristics has been addressed by several researchers [Moss et al. 1998; Stephenson et al. 2003] who advocate the use of Machine Learning (ML) as a methodology to automatically construct optimization heuristics [Wang and O'Boyle 2009]. Such an approach has the advantage of being portable across different platforms without requiring expert knowledge. However, until now, it has been limited to relatively straightforward problems where the target optimization to predict is fixed, for example, determining compiler flag settings [Hoste and Eeckhout 2008], loop unroll factors [Stephenson and Amarasinghe 2005], or the number of threads per parallel loop [Wang and O'Boyle 2009]. Determining the best partitioning of a stream program is fundamentally a more difficult task. First of all, rather than predicting a fixed set of optimizations we are faced with predicting an unbounded set of coalesce and split operations on a program graph. As the graph changes structure after each operation, this further increases the complexity. A secondary issue limiting the applicability of ML is its reliance on sufficient training data. This problem is particularly acute for emerging parallel programming languages where the application code base is small as is the case for streaming languages.

This article tackles both these problems. It develops a technique to automatically derive a good partitioning for StreamIt [Thies et al. 2001] programs on multicores making no assumption on the underlying architecture. Rather than predicting the best partitioned graph, it develops a nearest-neighbor-based Machine Learning (ML) model that predicts the ideal partitioned structure of the StreamIt program. It then searches through a program transformation space (without executing any code) to find a program of the suitable structure. To overcome the problem of insufficient training programs we have developed a microkernel stream program generator. This generator is able to provide many small training examples for the predictive model.

To show the automatic portability of our approach, we have evaluated our ML-based approach on two different multicore platforms. On a 4-core machine, on average, our approach achieves 1.90 times speedup over the dynamic-programming-based StreamIt partitioner, which translates to $60 \%$ of the performance gained by exhaustively evaluating over 3000 different partitions per program and selecting the best. Compared to a state-of-the-art analytical model-based approach, we achieve a $1.77 \times$ performance improvement. When ported to an 8 -core machine, we achieved $1.80 \times$ performance improvement over the the dynamic-programming-based StreamIt partitioner. 


\section{BACKGROUND AND MOTIVATION}

\subsection{Streamlt Language}

StreamIt [Gordon et al. 2002] is a language supporting streaming programming, based on the Synchronous DataFlow (SDF) model [Lee and Messerschmitt 1987]. StreamIt provides a set of language constructs to describe streaming parallelism and has an accompanying compiler and benchmarks, making it suitable for the work presented in this article.

In StreamIt, computation is performed by filters which are the basic computational units. A filter is a single-input, single-output block with a user-defined function which performs computation on the input item and translates the input to the output. Filters communicate through dataflow channels, which are implemented as FIFO queues. StreamIt provides a simple means of constructing rich hierarchically parallel structures such as pipeline and split-join. A StreamIt program often contains three types of coarse-grain parallelism: task, data, and pipeline parallelism. They are implemented in the following ways. Task parallelism is implemented as different branches in a split-join section, where each branch contains a number of filters and the output of each branch is never used by the other. Data parallelism is achieved by breaking up a "stateless" filter (a filter is "stateless" if there are no data dependencies between any two executions of the filter) into multiple filters that can be executed in parallel. Pipeline parallelism is implemented as multiple chains of filters that are directly connected in a StreamIt program.

Each StreamIt program is represented by a hierarchical stream graph. Each node of the stream graph is a task that can be executed in pipeline fashion. Concurrent task parallelism is achieved after each splitter node. Communication between tasks is defined by and restricted to the arcs between nodes. It is the compiler's responsibility to partition the stream graph and allocate partitions to threads which are then scheduled on the underlying hardware. This is a 2-stage process and is illustrated in Figure 3. First, the nodes in the original graph are merged into larger nodes or spilt into smaller nodes by a sequence of fuse and fission operations. This gives a transformed program where each node is allocated to a separate thread. Each thread is then scheduled to the hardware by the operating system. The first stage we call partitioning as it is concerned with determining those regions of the stream program that will be eventually allocated to a thread. The second stage we call scheduling and is responsible for the allocation of threads to processors. For example, Figures 15(b) and (c) are two different partitioning results to the FM benchmark (Figure 15(a)). Each node of the final partition will be mapped to a thread by the StreamIt backend compiler. In this article, we are interested in the mapping of nodes to threads and hence focus purely on the first stage of the process, ${ }^{1}$ partitioning.

\subsection{Motivation}

Finding a good partitioning for a streaming program is difficult due to the large number of possible partitions. Figure 2(a) illustrates a simplified stream graph for the MP3DECODER benchmark. Figures 2(b) and (c) show two possible partitioned versions of the original MP3DECODER program shown in Figure 1(a). Both are obtained by applying a sequence of fuse and fission operations on the original graph. The first partitioning shown in Figure 1(b) corresponds to a greedy partitioner, the second partitioning, Figure 1(c) corresponds to a dynamic-programming-based method; both are

\footnotetext{
${ }^{1}$ Note: as we use a machine learning approach, we implicitly consider the behavior of the thread scheduling policies along with the rest of the underlying system (hardware, operating systems, etc.) when generating training data.
} 


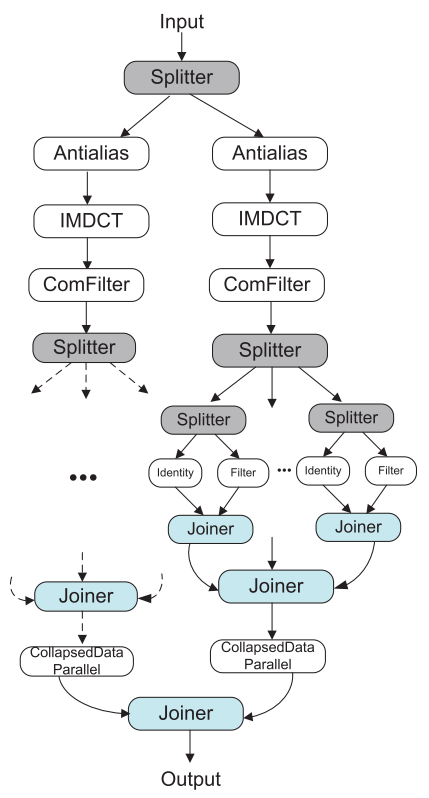

(a)

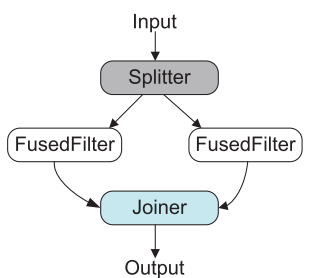

(b)

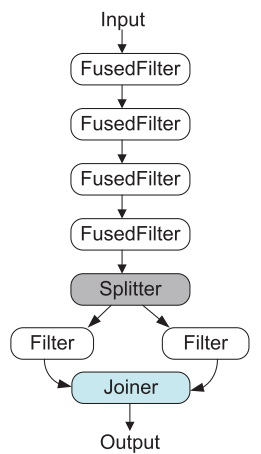

(c)

Fig. 1. A simplified stream graph of the MP3DECODER StreamIt program (a). Each node is a task that can be executed in pipeline fashion. Concurrent task parallelism is achieved after each splitter node. A greedy partitioner applied to this program gives the graph (b) with just 4 nodes. A dynamic-programming-based partitioner gives the graph (c) with 8 nodes.

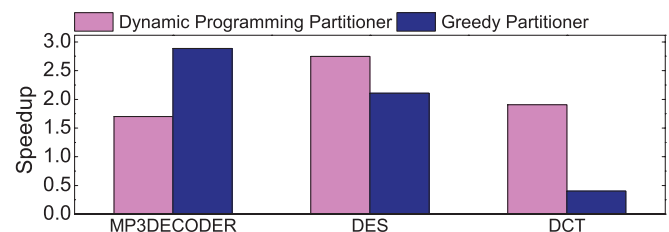

(a) 4-core platform

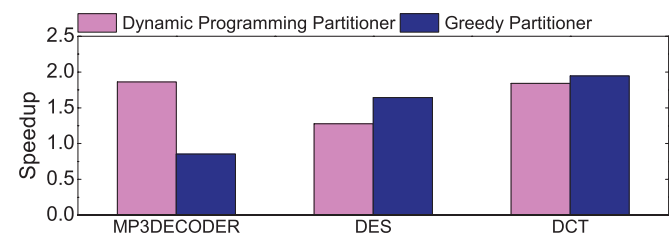

(b) 8-core platform

Fig. 2. The relative performance of 2 partitioning schemes with respect to a naïve partitioning scheme. The results are shown for 2 platforms and 3 distinct StreamIt programs. Greedy partitioning performs well on MP3DECODER on the 4-core platform but not as well as dynamic programming partitioning on the 8-core. This relative ordering is reversed for DES and DCT. Determining the best partitioning depends on program and platform.

StreamIt compiler built in heuristics [Thies et al. 2001]. The question is which is the best one? This problem of graph partitioning in its general form is known to be NP-complete and it is difficult to devise a general heuristics [Bui and Jones 1992].

To illustrate this point, consider Figure 2, which shows the performance of each partitioning approach on two different multicore platforms: a $2 \times$ dual-core (4-core) machine and a $2 \times$ quad-core (8-core) machine. On the 4-core the greedy scheme performs well for MP3DECODER; it has a lower communication cost, exploiting data parallelism rather than the pipeline parallelism favored by the dynamic programming partitioner. On the 8-core platform, however, the dynamic-programming-based heuristic delivers better performance as load balancing becomes critical.

When examining two further programs, DES and DCT, we see the best partitioning algorithm for a particular machine is reversed. The figure shows that there is no current 


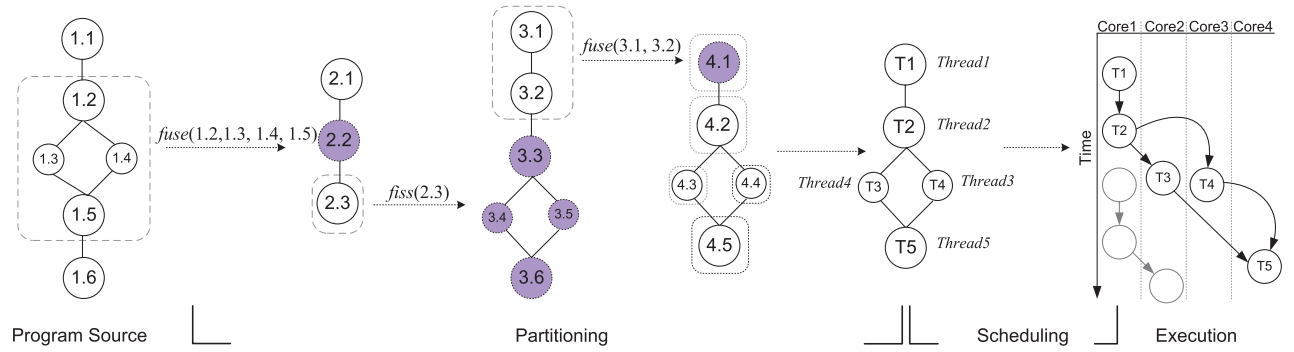

Fig. 3. The mapping process can be broken into 2 main stages: partitioning and scheduling. Partitioning is responsible for mapping nodes to threads. This is achieved by a series of fuse and fission operations on the original source program. At the end of this process, each node of the final graph is allocated to a thread and so the original graph has been partitioned. Scheduling allocates each of these threads to cores. Scheduling may be dynamic, especially if the number of threads is greater than cores. We focus solely on partitioning.
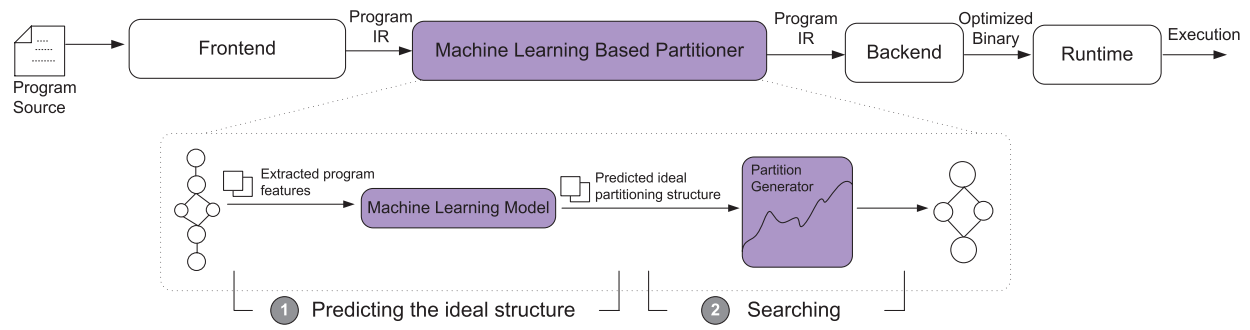

Fig. 4. Work flow of our compiler framework. The compiler takes in program source code and produces an optimized binary. In the middle of the compiler is a machine-learning-based partitioner. The partitioner first predicts the features of an ideal partitioning structure of the input program. This is done by checking the similarity of the input program features to prior knowledge. Then, it searches the transformation space to generate a program whose features are as close as possible to the predicted ideal structure.

"one-fits-all" heuristic and the best heuristic varies across programs and architectures. Rather than relying on heuristics, we would like a scheme that automatically predicts the right sequence of fuse and fission operations for each program and architecture. In the case of MP3DECODER, this means we want to select the operations that give the partitioned code in Figure 1(b) for 4 cores and the partitioned code in Figure 1(c) for 8 cores. However, predicting the correct sequences of fuse and fission is highly nontrivial given the unbounded structure of the input program graphs.

In the next section we describe our novel approach. Rather than predicting the sequence of fuse and fission operations directly, it tries to predict the right structure of the final partitioned program. Given this target structure, it then searches for a sequence of fuse and fission operations that generates a partitioned program that fits the predicted structure as closely as possible.

\section{PREDICTING AND GENERATING A GOOD PARTITION}

One of the hurdles in predicting the best sequence of fusion and fission operations (i.e., a sequence that leads to the best partition) is that the graph keeps changing structure after each operation. In Figure 3, the second operation fiss $(2.3)$ would have to be renamed fiss(1.6) if the first operation (fuse $(1.2,1.3,1.4,1.5)$ ) had not taken place. Any scheme that tries to predict a sequence of fuse and fission operations has therefore to take into consideration the structure of the graph at each intermediate stage. The supervised predictive modeling schemes explored to date are incapable of managing this [Duda et al. 2000]. We take a different approach. Instead of trying to predict the sequence of fuse and fission operations, we divide the problem into two stages as illustrated in Figure 4. 


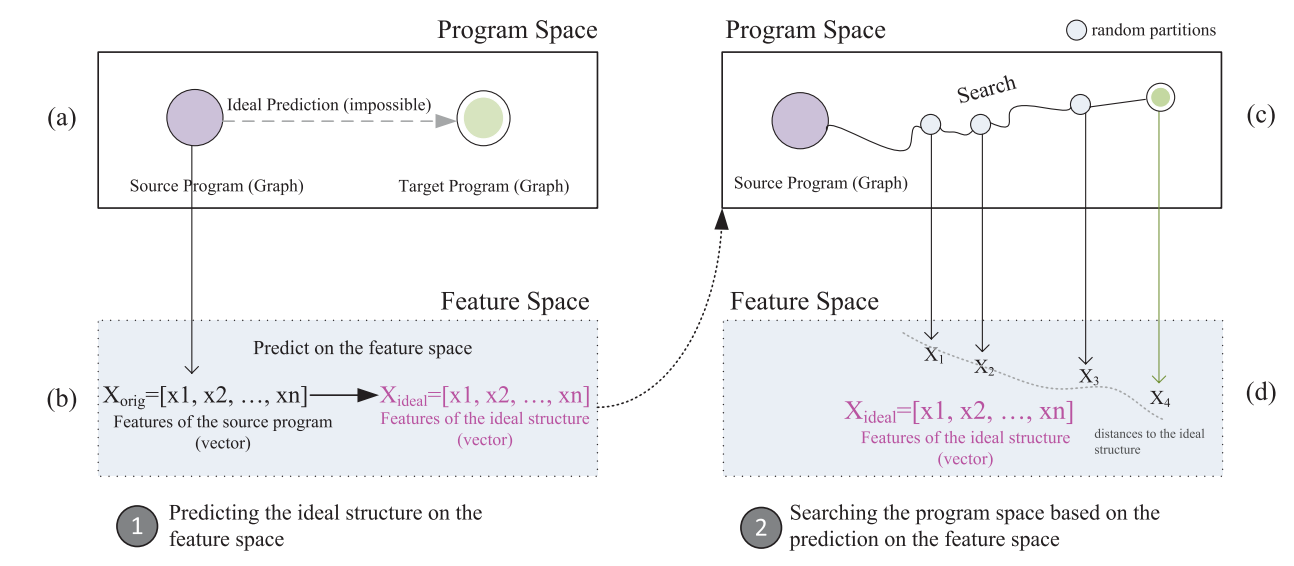

Fig. 5. The ML-based approach performs on two different spaces. It first predicts the feature vector of the ideal partitioning structure on the feature space. Then it searches the program space to generate a partition which is as close as possible to the ideal structure. The distance to the ideal structure of a generated partition is measured on the feature space.

(1) Predict the ideal structure of the final partitioned program.

(2) Search a space of operation sequences that delivers a program as close as possible to the ideal structure.

We use Figure 5 to explain the basic idea of our approach. The input program and the idea structure of the program are characterized by a fixed set of numerical values, called called features (see Section 3.2). The set of features is called a feature vector (e.g., $X$ in Figures 5(b) and (c)). Our 2-step approach actually performs on two different spaces. On the program space (Figure 5(a)), an ideal predictor would take in the input program graph and directly predict the output program graph. However, it is currently impossible to predict unbound graph structures using supervised learning. Instead our ML-based approach overcomes the problem by making predictions on the feature space first (Figure 5(b)) and it then maps the predicted result back to the program space (Figure 5(c)). In the first step the ML-based model predicts the ideal partitioning structure on the feature space (Figure 5(b)). It takes the feature vector of the input program and predicts the feature vector of the ideal program structure. On the feature space the prediction is performed on fixed feature vectors, which enables us to build a supervised learning model (see Section 3.1). Unfortunately there are no functions that can map the predicted feature vector back to a legal partition on the program space. To generate an output program, in the second step our approach first performs random search on the program space to generate a number of legal partitions (Figure 5(c)). It then measures the distance to the ideal structure (i.e. $X_{\text {ideal }}$ ) for each generated partition on the feature space (Figure $5(\mathrm{~d})$ ) and selects one partition whose distance is as close as possible to the ideal structure.

In essence, the first stage focuses on determining the goal of partitioning, that is, the structure of the partitioned program without regard to how it may be actually realized. The second stage explores different legal operation sequences until the generated partitioned program matches the goal. This frees us from the concern of correctly predicting the syntactically correct sequence of fuse and fission operations. Instead we can try arbitrary sequences until we reach a partition that closely matches our goal. The next section describes how we can predict a good partitioning goal and is followed by a section describing how we can generate a sequence of fuse and fission operations to reach that goal. 
Table I. Program Features Extracted from a Streaming Application

\begin{tabular}{ll}
\hline & Program Features \\
\hline \#Filter & \#Joiner \\
Pipeline depth & Split-join width \\
Avg. unit work & Max unit work \\
Pipeline work & Split-join work \\
Computation & Computation of stateful filters \\
Branches per instruction & Load/store per instruction \\
Avg. dynamic rate & Max dynamic rate \\
Avg. commun. rate & Computation-commun. ratio \\
Avg. commun. / unit work & Avg. bytes commun. / unit work \\
Max commun. rate / unit work & Work Balance \\
\hline
\end{tabular}

\subsection{Predicting the Ideal Partitioning Structure - Setting the Goal}

We wish to predict the ideal partitioned structure of any input graph program. In order to cast this as a machine learning problem, we wish to build a function $f$ which, given the essential characteristics or features $X_{\text {orig }}$ of the original program, predicts the features of the ideal partitioned program $X_{\text {ideal }}$ (as shown in Figures 5(b)). Building and using such a model follows the well-known 3-step process for supervised machine learning [Bishop 2006]: (i) generate training data, (ii) train a predictive model, (iii) use the predictor. We generate training data by evaluating (executing) randomly generated partitions for each training program and recording their execution time. The features of the original and best partitioned program are then used to train a model which is then used to predict the best ideal partitioning structure for any new unseen program. One of the key aspects in building a successful predictor is developing the right program features in order to characterize the original and goal program. This is described in the next section. This is followed by sections describing training data generation, building the predictor using nearest neighbors, and then using the predictor.

\subsection{Extracting Features}

Rather than trying to deal with unbounded program graphs as input and outputs to our predictor, we describe the essential characteristics of such graphs by a fixed feature vector of numerical values. The intention is that programs with similar feature vectors have similar behavior. We empirically evaluate this assumption in Section 7.2. In this work, we use program features to characterize a streaming application. The set of program features are summarized in Table I. We selected features that are important for performance from a programmer's perspective. All the work is statically estimated by the StreamIt compiler. Split-join width is the number of concurrent filters (i.e., work threads) in a split-join section. Unit work represents the amount of statically estimated work per iteration. Computation is the total amount of statically estimated instructions (by excluding instructions of pop and push operations) for all filters during the whole program execution. The communication rate represents the number of items popped and pushed by filters. Work balance is calculated by averaging the difference of estimated work across all pipeline stages.

We extract two sets of those features from the overall stream graph and the critical path of the program. Thereby, one set represents the overall characteristics of a streaming program and the other captures characteristics solely of the program's critical path. In other words, the whole feature vector consists of $2 N$ features $(N$ is the number of program features described in Table I) where $N$ features are extracted from the whole streaming graph and the other $N$ features are extracted from the critical path only. Features are extracted from the stream graph (i.e., program IR) without running the program, thus the overhead of extracting features is insignificant. 
For a given streaming program, our model first extracts a feature vector, $X_{\text {orig }}=$ $\left[x_{\text {orig }}^{1}, x_{\text {orig }}^{2}, \ldots, x_{\text {orig }}^{n}\right]$ from the original stream graph. $X_{\text {orig }}$ is used to characterize it. We use the same feature set $X_{\text {part }}$ to characterize any partitioning of a given program. We normalize the features and use Principal Component Analysis (PCA) [Bishop 2006] to reduce redundancies between features.

\subsection{Generating Training Data}

Once we have a means of describing the original and partitioned programs, we can start generating training data. Training data are generated by evaluating on average 3000 different randomly generated partitions for a program and recording the execution time, $t$. For each program, we also extract program feature sets, $X_{\text {orig }}$ and $X_{\text {part }}$, of both the program and its partition respectively. Program features and execution time are put together to form an associate training dataset $T=\left\{\left(X_{\text {orig }}^{i},\left(X_{\text {part }}^{i, j} t^{i, j}\right)\right\} i \in 1, \ldots, N\right.$ and $j \in 1, \ldots, R$, for $N$ training programs in which each program has $R$ different partitions.

One advantage of machine learning is that the learning algorithm automatically captures the impact of the thread scheduling policy from training data and implicitly correlates the scheduling policy with the best found partitions.

Synthetic Stream Program Generation. One particular problem encountered in training for new languages is that the training set is small. There simply is not a large enough program base with to work. To overcome this problem, we build a microkernel stream program generator to generate many small training examples, supplementary to existing benchmarks. This allows us to train our model on larger datasets. Our stream program generator automatically extracts microkernels (i.e., working functions and communicating patterns) from any subset of existing StreamIt programs. It limits the generated programs to a space with parallel parameters (i.e., pop and push rate, pipeline depth, split-join width, and loop iteration counts). Then, it generates a large number of small training examples in which the parallel parameters and working functions are varied.

The cost of generating new benchmarks can be neglected because millions of programs can be generated in an order of minutes. Running programs to generate training data from potentially thousands programs, however, is prohibitively expensive. Therefore, we select a limited number of representative programs by using a clustering technique that is also used to choose the ideal partitioning structure (as described in Section 3.4.1). Informally, we do this by examining the features of the programs generated and selecting only those which are sufficiently distinct from the existing training set. Although producing training data takes time, it is only a one off cost incurred by our model. Furthermore, generating and collecting data is a completely automatic process and is performed offline. Therefore, it requires far less effort than constructing a heuristic by hand.

\subsection{Building a Model}

Once we have generated sufficient training data, we are in a position to build a predictive model. Our model is based on a straightforward nearest-neighbor classifier [Duda et al. 2000]. For each training program, we record the features of the original program $X_{\text {orig }}$ and those of its best found partition $X_{i d e a l}$. When used on a new unseen program, we find the program from the training set whose features most closely match the new program's features. We then simply return the features $X_{i d e a l}$ of the training program as the predicted best ideal partition for the new program.

In our setting, each program in fact has a number of partitions that give good performance. To capture this we group the best partitions into regions using clustering. 
We consider the cluster with the best average performance and select a representative candidate as described in the next section.

3.4.1. Selecting the Ideal Partitioning Structure. Each training program has a number of good partitions. Our task is to select the most useful one, that is, the partition that is likely to be good for similar programs. We cluster the good partitions using a $k$-means clustering algorithm [Bishop 2006]. In order to determine the right number of clusters $K$, we use the standard Bayesian Information Criterion (BIC) score [Pelleg and Moore 2000; Schwarz 1978] to decide how many clusters should be selected. BIC is a measurement of the "goodness of fit" of a clustering parameter (i.e., $K$ ) to a given dataset. The larger the BIC score, the higher chance that we find a good clustering number for the dataset. We use the BIC formulation given in Pelleg and Moore [2000], which is

$$
B I C_{j}=\hat{l}_{j}-\frac{p_{j}}{2} \cdot \log R
$$

where $\hat{l}_{j}$ is the log-likelihood of the data when $K$ equals to $j, R$ is the number of points in the data (i.e., the number of generated partitions), and $p_{j}$ is the number of free parameters to estimate, which is calculated as: $p_{j}=(K-1)+d K+1$ for a $d$-dimension feature vector plus 1 variance estimate [Sherwood et al. 2002]. $\hat{l}_{j}$ is computed as

$$
\hat{l}_{j}=\sum_{n=1}^{K}-\frac{R_{n}}{2} \log (2 \pi)-\frac{R_{n} \cdot d}{2} \log \left(\hat{\sigma}^{2}\right)-\frac{R_{n}-K}{2}+R_{n} \log \left(R_{n} / R\right)+\log S_{n},
$$

where $R_{n}$ is the number of points in the $n$th cluster, $\hat{\sigma}^{2}$ is the average variance of the distance from each point to its cluster center, and $S_{n}$ is the normalized average speedup of the $n$th cluster.

We apply the $k$-means clustering algorithm for the generated programs by varying the cluster number $K$. For each clustering result, we first calculate its corresponding BIC score. We choose a clustering number, $K_{\text {best }}$, which gives us the highest BIC score. After this point, we know the partition space can be represented by $K_{\text {best }}$ clusters.

Once we have found the number of clusters, we select the cluster that has the largest number of good partitions. We select the $10 \%$ of partitions that are close to the cluster centriod of the selected cluster and normalize their feature values. The normalized features are considered as the ideal partition structure.

Figure 6 visually depicts the use of this clustering technique. Each point in the figure represents a good partition of the StreamIt benchmark LATTICE. To aid clarity, we have projected the dimension of the feature space down to 2 . For these programs there are 3 distinct clusters of good partitions. Cluster- 1 is chosen because it contains $66 \%$ of all the partitions and has the highest mean speedup.

3.4.2. Selecting Representative Benchmarks. Our synthetic benchmark generator can generate millions of different StreamIt benchmarks in an order of minutes. Executing a potential large number of training programs, however, is very expensive. To build an accurate model in an affordable time, we use a similar $k$-means algorithm as presented in Section 3.4.1 to select the most representative programs for training. Similar to the method that is used to select the ideal partitioning structure, we first use $k$-means algorithm to group the synthetic benchmarks into a number of clusters and then select a few programs from each cluster as representative programs. The number of clusters is determined by the BIC score that is defined by Eq. (1). $\hat{l}_{j}$ of Eq. (1) is calculated by

$$
\hat{\imath}_{j}=\sum_{n=1}^{K}-\frac{R_{n}}{2} \log (2 \pi)-\frac{R_{n} \cdot d}{2} \log \left(\hat{\sigma}^{2}\right)-\frac{R_{n}-K}{2}+R_{n} \log \left(R_{n} / R\right),
$$




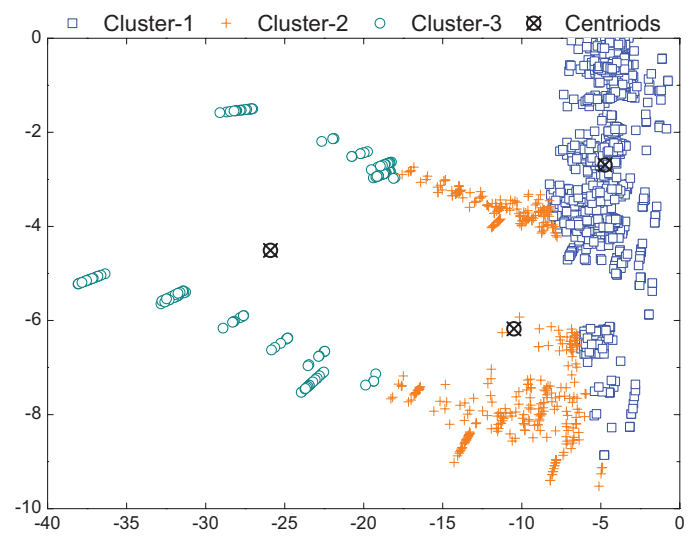

Fig. 6. The feature space is projected into 2 dimensions in this diagram for presentation purposes. Each of the points represents a good partitioning structure for the LATTICE program. Using clustering there are 3 clusters found, where the center of each is marked as the centroid. Cluster- 1 is selected as it has the best mean speedup. The features around the centroid of cluster-1 are then averaged and used as the ideal partitioning structure.

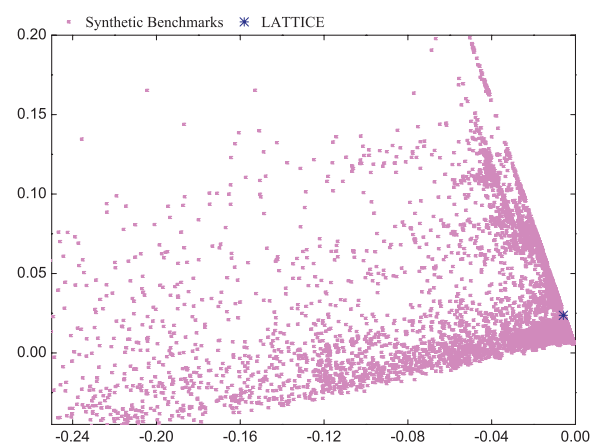

(a) synthetic programs

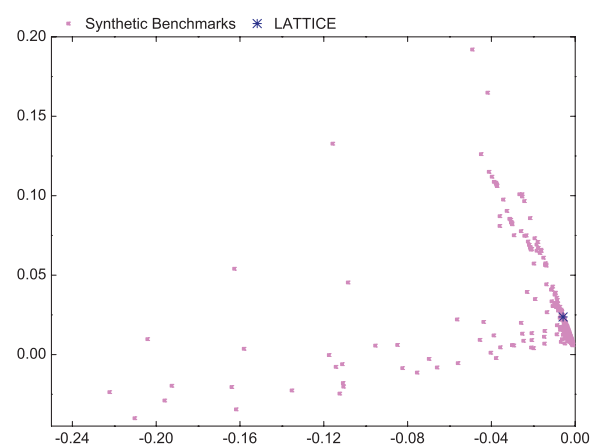

(b) representative benchmarks

Fig. 7. Using the benchmark generator to generate representative training benchmarks.

where $R_{n}$ is the number of points in the $n$th cluster and $\hat{\sigma}^{2}$ is the average variance of the distance from each point to its cluster center.

We apply the $k$-means algorithm to the generated programs with different $K$. For each clustering result, its corresponding BIC score is computed. The clustering result that gives the best BIC score is chosen. We then select $1 \%$ of programs from each cluster as representative programs.

The process of synthetic benchmarks generation is visually depicted in Figure 7. In this example LATTICE is excluded from the training program set. It is desired to have a training program set that is compact while sufficient enough for building an accurate predictor, that is, the training program set should consist of programs whose features are similar to LATTICE. To achieve this goal the benchmark generator first extracts microkernels from the available training programs (where LATTICE is excluded) and uses the extracted small StreamIt kernels to generate many new, small training examples as shown in Figure 7(a). We then use the $k$-means algorithm to select representative programs. For this example we selected around 200 synthetic programs as shown in Figure 7(b). As can be seen from the figure, the selected benchmarks fully 


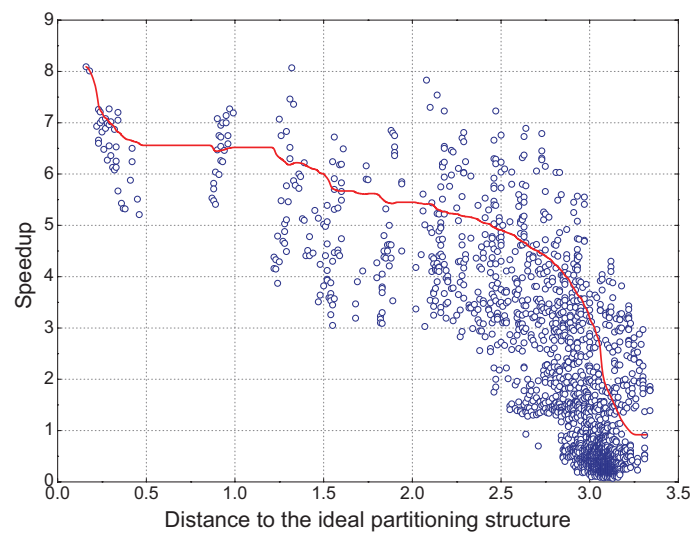

Fig. 8. Separating partition candidates with Euclidean distances. Each dot represents a partition choice and the line represents the mean speedup of that partition within a distance.

cover the unseen program, namely LATTICE, in the feature space. Using the synthetic programs, the resulting model gives accurate predictions for LATTICE.

\subsection{Using the Model to Predict the Ideal Partitioning Structure}

Once we have gathered training data and built the model as described before, we can now use the model to predict the ideal partition structure of a new, unseen program as shown in Figure 4. We firstly extract features of the input program, normalize its program features using PCA, and use the nearest-neighbor model to predict the ideal partitioning structure of the input program. The nearest-neighbor scheme picks a program in the training set that is the most similar to the input program. This is done by comparing the input program's features to known programs' features. Once the nearest neighbor has been selected, we use its ideal partitioning $X_{\text {ideal }}$ as the predicted ideal structure for the new program. In rare instances, our model may not be able to find any training programs that are similar enough to the input program (i.e., no programs in the training set are close to the new input program). It then simply uses the default partitioner provided by the compiler. We empirically determined the similarity threshold. A training program is not considered to be close enough if its Euclidean distance to the input program is greater than 10.5 .

\section{SEARCHING AND GENERATING A PARTITION CLOSE TO THE PREDICTED IDEAL STRUCTURE}

The previous section provides a means to predict the ideal partition structure without actually running the code. We now generate new partitions by applying random fuse and fission operations to the input program's graph. For each generated partition, we measure its Euclidean distance from the predicted ideal structure in the feature space. We repeat this many times, selecting the partition nearest the ideal structure.

Figure 8 illustrates the use of distance as a means of determining the best partition candidate for the StreamIt program LATTICE. Each dot represents a unique partition. There are over 3000 different partitions of which only $15 \%$ partitions are better than the partition generated by the StreamIt default scheme. Given the large number, selecting a partition to improve is nontrivial. The figure shows that distance to the ideal structure is a good measure of the quality of a partition. If we choose a distance of less than 0.5 as the confidence level, then we will pick a partition that is at least 5.21 times (6.6 times 
on average) faster than a partition generated by the StreamIt default partitioning heuristic.

For the purposes of this article, we generate on average 3000 potential partitions for each new program, selecting the one that is nearest to the ideal. We do not execute any of these programs, merely extract their features and measure the Euclidean distance. Each partition takes less than $100 \mathrm{~ms}$ to generate and evaluate, so is not a significant cost.

\section{EXPERIMENTAL METHODOLOGY}

This section describes the platforms, compilers, and benchmarks used in our experiments as well as the evaluation methodology.

Benchmarks. We use the StreamIt benchmark suite to evaluate our approach. These applications represent typical streaming parallel programs containing task, data, and pipeline parallelism. On average, each program contains 46 (up to 168) filters at IR level. The MPEG2 benchmark has filters with dynamic rates, that is, the exact number of items produced by a filter, and the amount of computation on any given execution is unknown at compile time.

Input Data. We used the standard StreamIt benchmark input data to evaluate our approach. The current StreamIt compiler implementation imposes a restriction that the loop trip count must be statically known at compiler time. Because of this, the input data is hard-coded into the source code. This prevents us from evaluating one partition across different input datasets.

Compilers. We implemented our machine learning model as a stream graph partitioner in the StreamIt compiler (version 2.1.1). The StreamIt compiler is a source-tosource compiler which translates the partitioned stream graph to $\mathrm{C}++$ code. The Intel $\mathrm{C} / \mathrm{C}++$ compiler (ICC) version 11.0 was used to convert the $\mathrm{C}++$ program to binary. We use "-03 -xT -aXT -ipo" as the ICC compiler flags.

Hardware Platform. The experiments were performed on two multicore platforms: a 4-core platform (with two dual-core Intel Xeon 5160 processors running at $3.0 \mathrm{GHz}$ and has 8GB memory) and an 8-core platform (with two quad-core Intel Xeon 5450 processors running at $3.0 \mathrm{GHz}$ and has $16 \mathrm{~GB}$ memory). Both platforms run with 64-bit Scientific Linux with kernel 2.6.17-164 x86_64 SMP.

Cross-Validation. We use leave-one-out-cross-validation to evaluate our approach [Bishop 2006]. This means we remove the program to be partitioned from the training program set and then build a model based on the remaining programs. This also guarantees that our benchmark generator has not seen the target program before. The trained model is used to generate partitions for the removed target program. We repeat this procedure for each program in turn. It is a standard evaluation methodology, providing an estimate of the generalization ability of a machine-learning-based model in predicting for an unseen programs.

Synthetic Benchmarks. We generate roughly over $100 \mathrm{~K}$ synthetic programs and select around 60 for training. The benchmark generation and selection process takes less than an hour.

Feature Selection We use PCA to reduce the number of features without significantly losing variances (Section 3.2) of the raw features. We used principal components that contribute to $95 \%$ of the variances after applying PCA on the raw feature vector. The processed feature vector contains on average 10 principal components. 


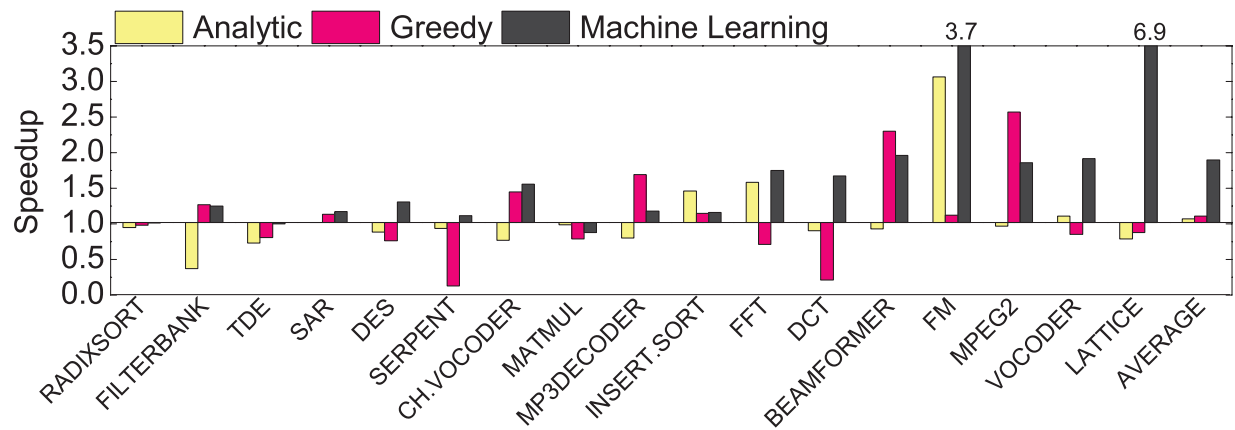

Fig. 9. Performance comparison on the 4-core platform for the analytical model, the greedy partitioner, and the ML-based model.

\subsection{Comparison}

StreamIt has its default partitioning strategy, a sophisticated dynamic-programmingbased partitioning heuristic [Thies 2009]. All results are presented relative to this default and it provides a challenging baseline. To provide a wider comparison, we also evaluate a recently proposed analytical-based pipeline parallelism model [Navarro et al. 2009] and an alternative greedy-based heuristic available within the StreamIt compiler [Gordon et al. 2006]. The analytical-based model finds a suitable parallel mapping by predicting the execution time of a given streaming application. We have implemented the analytical-based partitioner in the StreamIt compiler. For each program, the partitioner generates 50,000 partitions of a single program and selects a mapping which has the best predictive performance as output. Our scheme, in contrast, predicts the best structure and selects the partition closest to it, using an order of magnitude fewer candidates.

Best Performance Found. In addition to comparison with existing approaches, we wish to evaluate our model by assessing how close its performance is to the maximum achievable. However, it is not possible to determine the best, due to the combinatorially large number of partitions. Instead we randomly generated 3,000 different partitions for each program and select the best performing partition as an indication of the upper bound on performance that could be achieved if we had sufficient resources. We call this "best found" performance.

\section{EXPERIMENTAL RESULTS}

In this section we first report the performance of our approach against alternative schemes on the 4-core platform. This is followed by a short explanation of the results generated by different models. Next, we evaluate the accuracy of our model in predicting ideal structures. Finally, we extend our model to an 8-core platform and evaluate its performance.

\subsection{Performance Comparisons}

6.1.1. Comparison with other Techniques. Figure 9 shows the performance results for the 4-core platform. On average the analytical-based and the greedy-based partitioners do not significantly improve over the StreamIt default dynamic-programming-based partitioner. Our approach, however, is able to deliver significant improvement over the default scheme with a $1.90 \times$ average speedup.

Analytic. On average, the analytical-based model only achieves $1.07 \times$ speedup over the StreamIt default partitioner. This is not a surprising result because the StreamIt 


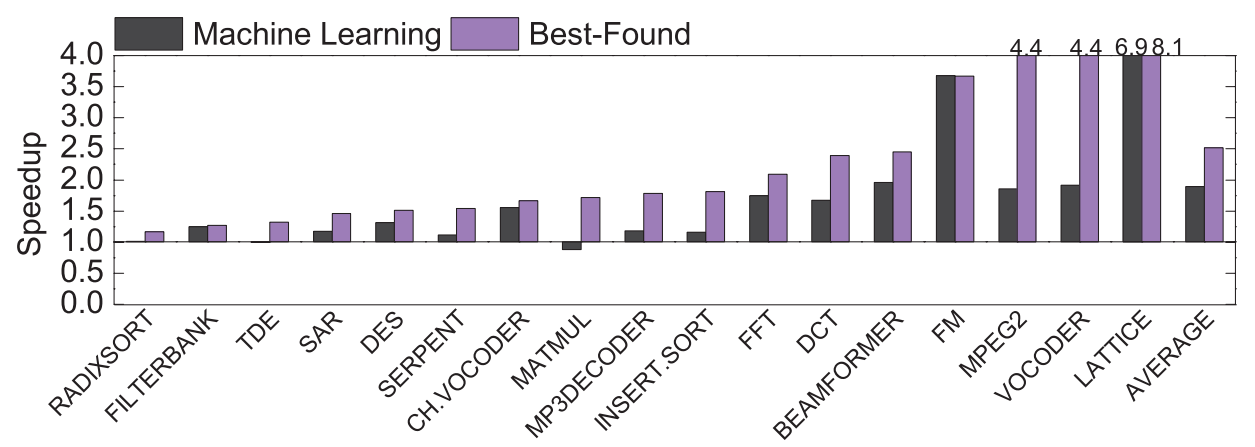

Fig. 10. The performance of our approach vs the best performance found out of on average 3000 executions per program on the 4-core platform.

default partitioner is a strong baseline tuned by hand. It is only able to improve performance in 4 out of the 17 programs. It successfully partitions FM resulting in a $3.0 \times$ speedup by coarsening the pipeline. However, it fails to balance the pipeline of FILTERBANK due to its inability to capture the complicated communication pattern of the program. This leads to a greater than $2 \times$ slowdown.

Greedy. The greedy partitioner also fails to significantly improve over the StreamIt default partitioner. On average, it achieves a $10 \%$ performance improvement over the default partitioner. In approximately half of the programs, it gives a performance improvement. For example, it is able to achieve an impressive $1.69 \times$ and $2.57 \times$ speedup on MP3DECODER and MPEG2 by reducing communication through aggressive fusion. However, it also slows down 9 applications, particularly in the case of SERPENT and DCT, which are up to $7.7 \times$ slower than the default dynamic programming approach. This result clearly shows the best partitioning heuristic varies from program to program.

Our approach. Our machine-learning-based approach, on the other hand, can greatly improve performance compared to the default partitioner and gives more stable results. It achieves better performance in most of the benchmarks, up to $6.9 \times$ for LATTICE. In just one case, MATMUL, we perform worse than the default (as do the other 2 schemes). The backend ICC compiler aggressively performs autovectorizaion on the program which has not been captured by our model. This issue can be solved by adding additional features to the model and is the subject of future work.

6.1.2. Comparison vs Best-Found Performance. Although our scheme performs well compared to existing approaches, it is useful to know whether there is further room for improvement (further discussion is provided in Section 7). In Figure 10, we compare our scheme against an approximation to the best found performance. For FM we reach this maximum, but for other programs such as MP3DECODER, INSERTIONSORT, and VOCODER, there is significant room for improvement. So although our approach outperformed all prior techniques on VOCODER, it could have done better. Overall there is a $2.5 \times$ average maximum speedup available and we achieve $60 \%$ of that maximum performance.

Figure 11 shows the throughput of our approach and the best found performance on the 4-core system. Throughput is calculated by counting the number of input items processed within a second. Overall, both approaches are able to process over $100 \mathrm{~K}$ items per second across all benchmarks. For some applications, such as VOCODER, FM, and DCT, high throughput was observed. For small applications, such as RADIXSORT, INSERTIONSORT, and BEAMFORMER, the communication overhead outweighs the 


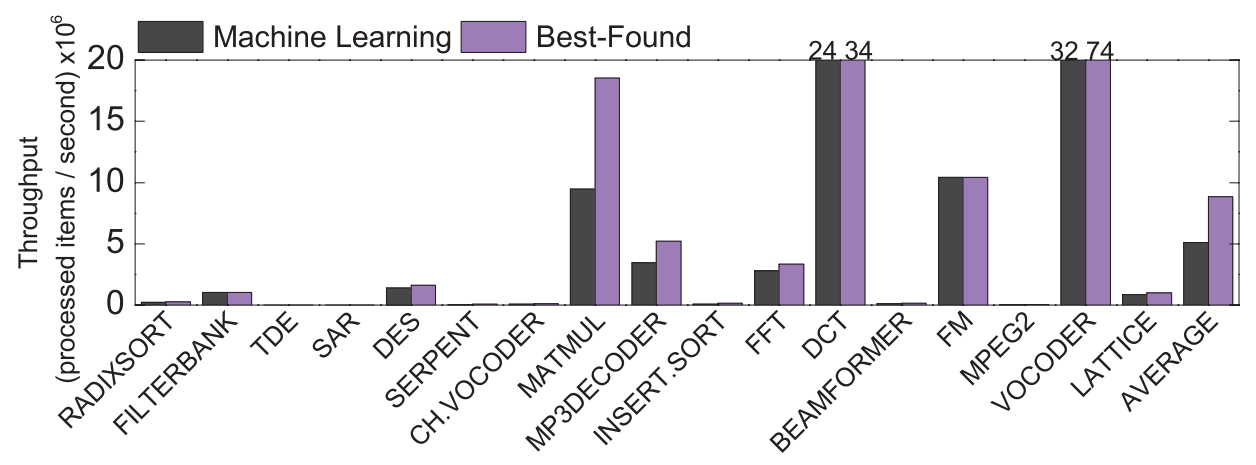

Fig. 11. Throughput of our approach vs the best performance found out of on average 3000 executions per program on the 4-core platform.

benefit of parallel execution, thus those benchmarks have relatively low throughput. For complex applications, such as TDE, SAR, and MPEG2, the achieved throughput is restricted by the fact that it takes longer for the pipeline to translate inputs to outputs than other applications. Overall we achieve 58\% of that maximum throughput.

\section{EXPLANATION}

In order to get further insights into the different models, we investigate partitions generated by different approaches of five selected StreamIt benchmarks. This allows us to directly compare the ML-based approach on the applications that other approaches perform well. We also used the Intel VTune Amplifier XE 2011 profiling tool to analyze some of the benchmarks.

RADIXSORT. This application has a regular parallel structure: it is pure pipeline parallelism; 10 out of its 13 filters have exactly the same computation-communication ratio. For this program, both the dynamic-programming-based and the greedy-based algorithms give a partition that has the best found performance. Thus, our approach is not able to improve their results.

LATTICE. This application contains 36 filters with both data and pipeline parallelism as shown in Figure 12(a). Finding a good partition for it is certainly nontrivial and different approaches give different partitions. Figures 12(b) to (e) illustrate the partitions given by four partitioners: the StreamIt default, the greedy-based, the analytical-based, and our ML-based partitioners, respectively. The StreamIt default partitioner aims to form a balanced pipeline and generates a partition with four nodes. This partition outperforms the solutions given by the greedy-based and the analytical model-based partitioners, which generate partitions with more threads at the scheduling stage bringing runtime overhead. In contrast, our approach generates a coarse-grain stream graph, which has relatively fewer number of threads and lower communication cost. As a result, the ML-based approach achieves better performance than other techniques. When compared to the 4-node partition given by the StreamIt default scheme (Figure 12(a)), our ML-based approach reduces the communication and synchronization overhead by a factor of 8 and pipeline stall overhead by a factor of 1.5. By examining this application, we discover that the computation of LATTICE is relatively small compared to the communication cost on the 4-core platform (as can be indicated by the relatively low throughput in Figure 11). Therefore, a good partitioning strategy will try to coarsen the stream graph to reduce communication overhead. In this case, our approach identifies the program characteristics of LATTICE and applies an appropriate heuristic to aggressively coarsen the stream graph. When compared 


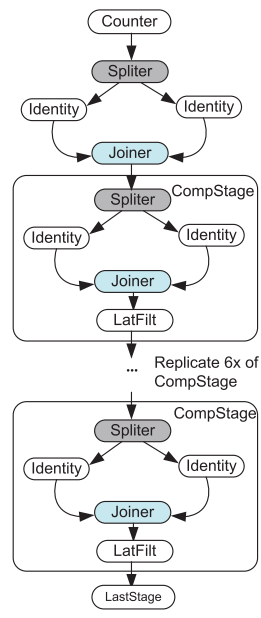

(a)

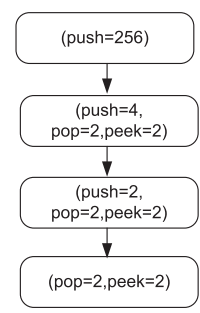

(b)

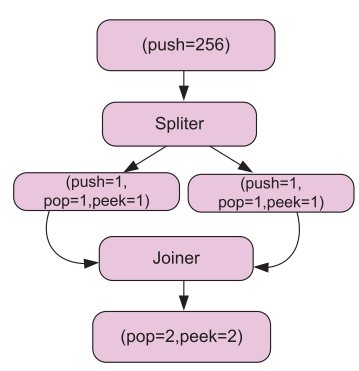

(c)

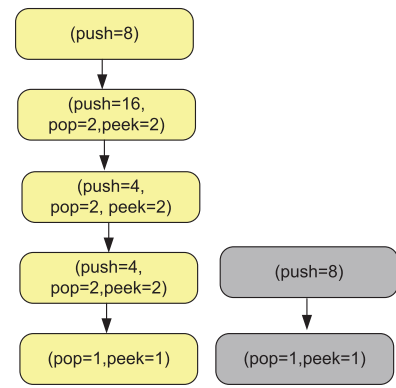

(d)

(e)

Fig. 12. The stream graph (a) and partitions generated by different approaches for LATTICE. Each box represents a filter and the communication rate of each filter is denoted. The dynamic-programming-based partitioner gives (b), the greedy partitioner gives (c), the analytical model gives (d), and our ML-based approach gives (e) by coarsening the stream graph to reduce communication overhead.

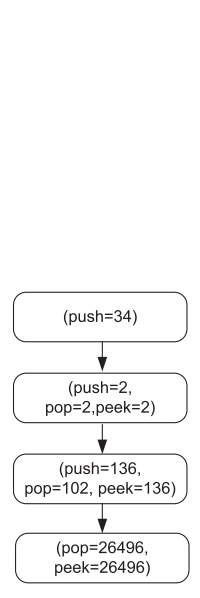

(a)

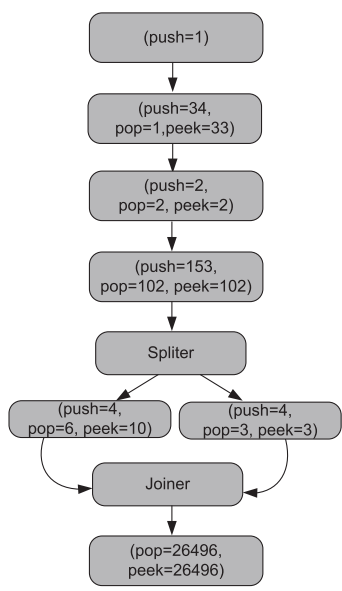

(b)

Fig. 13. Partitions generated by the StreamIt default method (a), our approach (b), and the "best found" partition (c) for VOCODER. The StreamIt default scheme exploits purely pipeline parallelism. Our ML-based approach exploits both pipeline and task parallelism.

to the StreamIt default partitioner, the partition generated by our approach reduces pipeline stalls by a factor of 3 .

VOCODER. The stream graph of VOCODER has over 120 filters containing large split-join sections and long-stage pipelines. Unlike LATTICE's partitioning strategy, merely considering coarsening the stream graph is not the right choice for this application. Figures 13(a) and (b) correspond to the partitions given by the StreamIt default scheme and our approach, respectively. This time, our ML-based approach takes a different strategy. In order to reduce communication overhead, it first coarsens those small computation kernels. At the same time, it exploits task parallelism in the critical path 


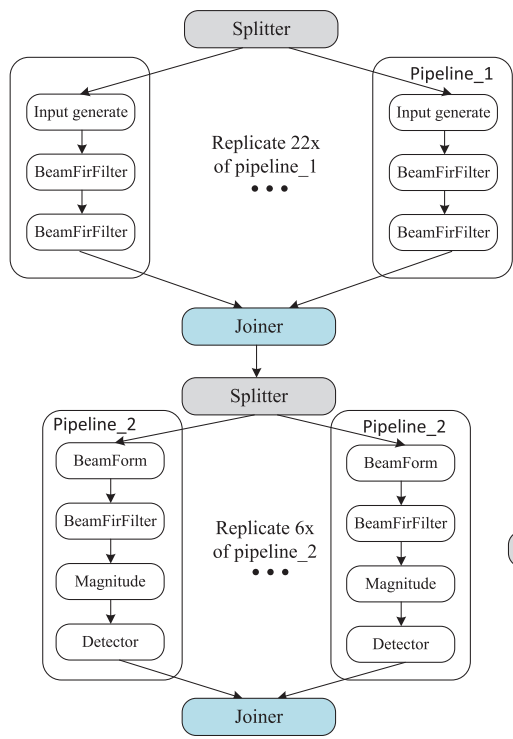

(a)

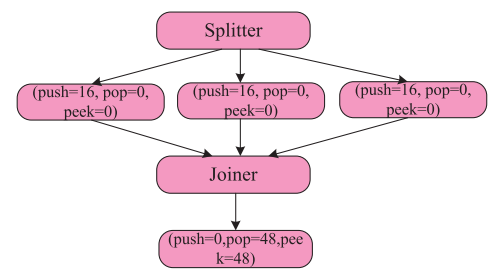

(b)

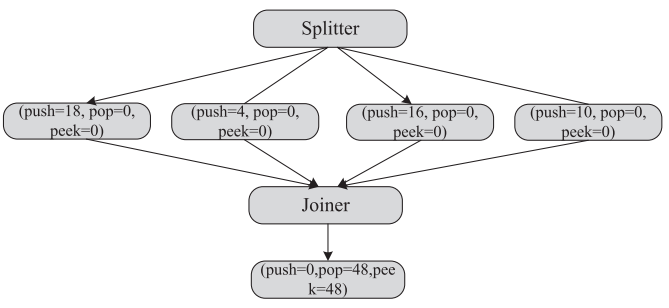

(c)

Fig. 14. Partitions of the original BEAMFORMER graph (a). The greedy-based partitioner gives (b) and our ML-based approach gives (c).

(which contributes to around $40 \%$ of the total computation) and generates a 9-node partition. When compared with the best found solution, a 13-node partition, the main split-join section does not fully utilize the parallel hardware. This leads to $2.8 \times$ longer stall time for the joiner thread. Our ML-based model could be smarter by predicting a more aggressive partitioning goal.

BEAMFORMER. This application consists of two split-join sections as shown in Figure 14(a). The greedy partitioner evenly distributes communication across filters of split-join sections (Figure 14(b)), achieving the best performance by exploiting task parallelism. Like the greedy-based partitioner, our model also predicts to exploit task parallelism, resulting in a program as shown in Figure 14(c). The reason that our approach performs less well than the greedy algorithm for this occasion $(2.0 \times$ rather than $2.3 \times$ speedups) is because the ML model does not evenly distribute communication across filters of the split-join section. As such, it leads to $30 \%$ of slowdown in the split-join section because of unbalanced work distribution among the four worker threads. This can be improved by adding an additional feature to characterize the work distribution across filters of major split-join sections.

FM. This application simulates a software FM radio (Figure $15(\mathrm{a})$ ). The input is processed by a demodulator to produce an audio signal which is then passed through an equalizer. The equalizer is implemented as a split-join section with a number of filters. Both the analytical scheme and our model decide to exploit pipeline parallelism for this application as shown in Figures 15(b) and 15(c) respectively. The analytical model achieves $3 \times$ speedups over the StreamIt default partitioner. Our model outperforms the analytical model by a factor of $1.23 \times$. This is achieved by balancing the communication and computation of the last two filters in the pipeline, that is, the final stage of the pipeline peeks 2 items instead of 256 items each time. 


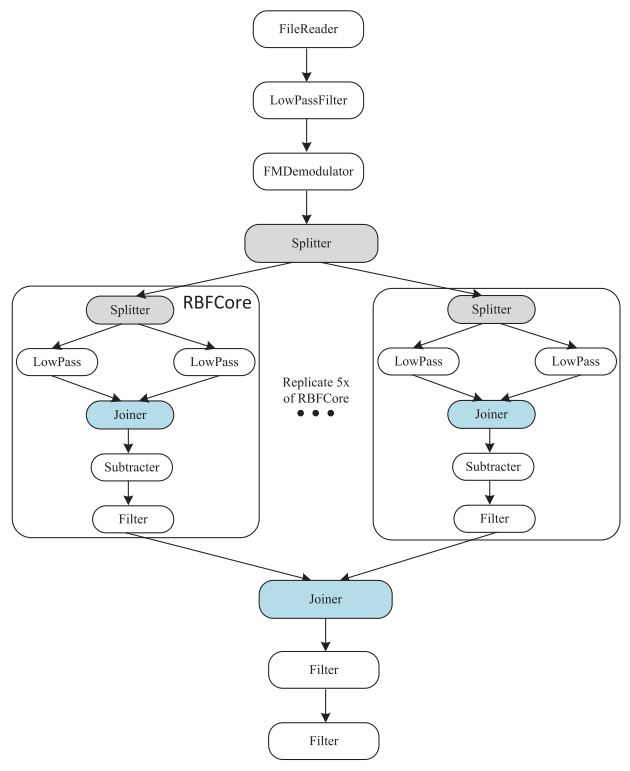

(a)

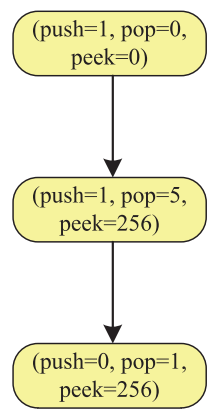

(b)

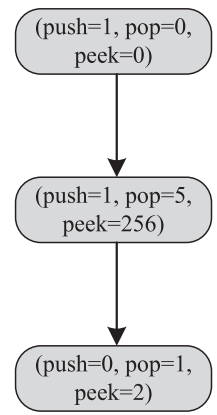

(c)

Fig. 15. Partitions of the original FM stream graph (a). The analytical model generates (b) and the ML-based model produces graph (c).

\subsection{Summary of Explanation}

As indicated by these examples, we can see that different partitioning strategies should be applied to applications with different program characteristics (Section 7.2.4 gives detailed discussion about the program characteristics). Essentially, the analytical model and the two StreamIt partitioners are "one-size-fits-all" strategies. They can be improved by a program-aware partitioning scheme. Developing such a scheme by hand is, however, extremely hard. Our approach, on the other hand, overcomes this problem by leveraging machine learning techniques. It uses prior knowledge to select and apply the program-specific partitioning strategy according to program characteristics of the target program, resulting in better performance.

\subsection{Analysis of Results}

This section provides analysis of our approach. We first compare the number of filters of the best found partitions. Next, we evaluate the accuracy of the nearest-neighbor model. This is then followed by an evaluation of how useful our feature space is in distinguishing good partitions. Finally, we examine the best found partitioning structures and what optimization criteria are important in delivering performance.

7.2.1. Best Found Numbers of Filters. Table II shows the number of filters of the best found partition for each application. When converting the generated partition to a binary, the StreamIt backend allocates each filter to a thread (i.e., pthread). For some applications such as FFT, FM, and DCT, the best found performance is only achievable by using fewer threads than the number of available physical cores (i.e., 4 cores). This is because the communication overhead of these applications is much higher than their computation. As a result, hiding the communication overhead by coarsening the stream graph is important for these applications. By contrast, it is more important to exploit parallelism for applications like SAR, MP3DECODER, and TDE; the best partitions 
Table II. Numbers of Filters of Best Found Partitions

\begin{tabular}{lclc}
\hline Program & \#Filters & \multicolumn{1}{c}{ Program } & \#Filters \\
\hline RADIXSORT & 5 & FILTERBANK & 12 \\
TDE & 13 & SAR & 16 \\
SERPENT & 10 & CHANNELVOCODER & 7 \\
MATMUL & 6 & MP3DECODER & 14 \\
INSERTSORT & 8 & FFT & 2 \\
DCT & 4 & BEAMFORMER & 8 \\
FM & 2 & MPEG2 & 11 \\
VOCODER & 10 & LATTICE & 3 \\
\hline
\end{tabular}

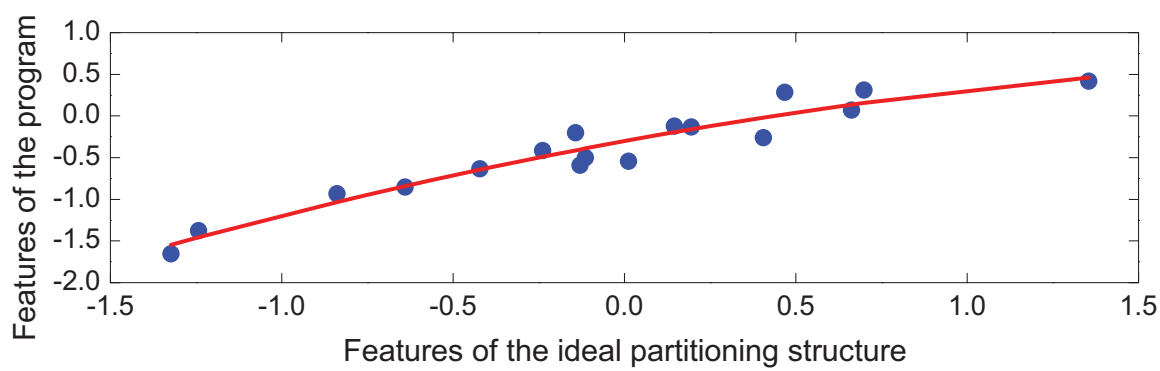

Fig. 16. Correlation between program features and the ideal partitioning structure. This figure shows there is strong correlation between program features of a program and its ideal partitioning structure. Note that the feature vector of each program has been reduced into a single value to aid visualization.

consist of 13 to 16 filters. Generating a high number of filters exposes parallelism of the application where the bottleneck benefits from parallel executions. For the rest of the programs, the best found partitions contain 4 to 12 filters. This table again confirms that the best partition varies from program to program and as a result, a fixed heuristic is not able to achieve stable performance across programs.

7.2.2. Correlation of Program Features. The intuition behind our predictive model is that similar programs will have similar ideal partitioning structures as long as we are able to have features that capture similarity accurately. This assumption can by confirmed by Figure 16. It shows the program features of the ideal partitioned program versus the features of the original program for each of the benchmarks. The original multidimensional feature vectors have been projected into a single value for each program to aid clarity. This figure shows a strong correlation between the program features and the ideal partitioning structure. We can quantify this by using the correlation coefficient [Bishop 2006]. It takes a value between -1 and 1 , the closer the coefficient is to $+/-1$, the stronger the correlation between the variables. It is 0.9 in our case , which indicates a high correlation between program features and the ideal partitioning structure. This means the premise for the nearest-neighbor model is valid.

7.2.3. Distance-Based Mapping Selection. The box plots in Figure 17 summarize the performance of partitions around a predicted ideal partitioning structure. It shows the performance of partitions with a normalized distance of less than 0.5 to the predicted ideal partitioning structure, as used by our scheme. The diagram shows that regions around the predicted ideal result in good performance. The top and the bottom of the "whisker" of each program represent the highest and the lowest speedup found in the region around the predicted ideal. For the majority of programs we obtain significant performance improvement if we can generate a mapping that is closer to the predicted ideal partitioning structure. The one exception is MATMUL, as seen in Figure 10. If 


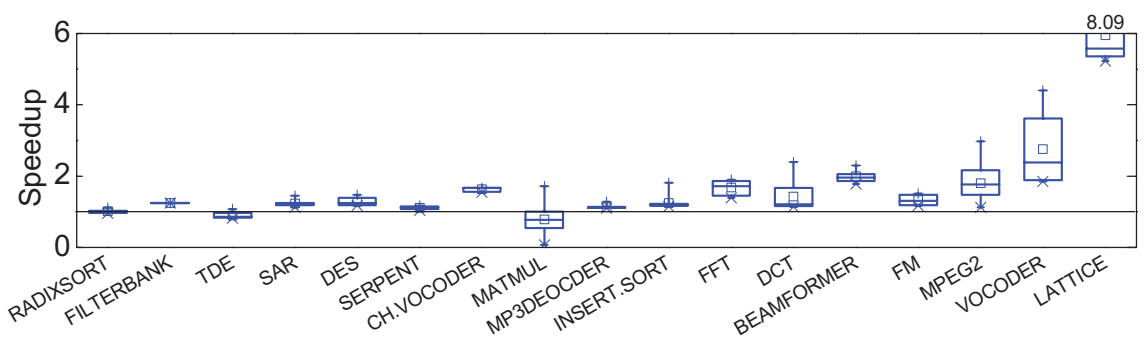

Fig. 17. Performance of mappings around the predicted ideal partitioning structure. The central box denotes the mean speedup and the top and bottom of each box represent the highest and the lowest speedup using our predictive model.

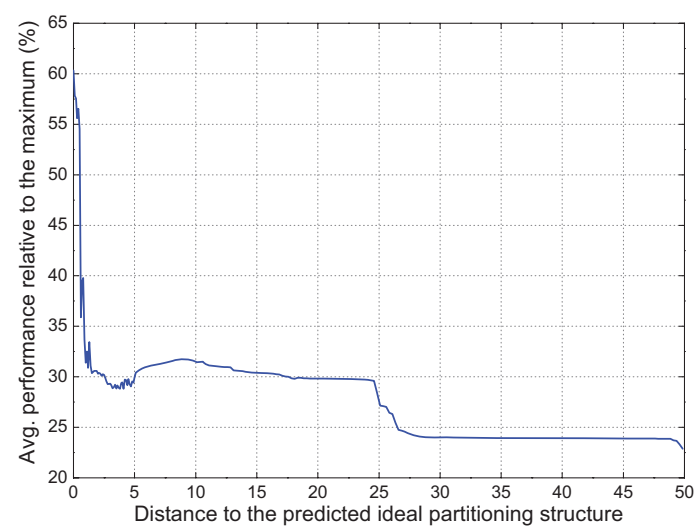

Fig. 18. Average performance relative to upper bound vs distance from ideal partition. This figure shows the average performance of a partition as a function of its distance from the predicted ideal structure. As the distance decreases, performance improves.

we zoom in on VOCODER, we see that the average speedup obtained in this region is 2.7. The lower value is 1.9 and the upper is 4.4. If we look at Figure 10, we see that our scheme selects a partition that achieves just a 1.9 speedup-the lower bound, while the best performance is 4.4 - the upper bound. This shows that our scheme could improve if it was smarter in choosing the ideal structure within a good cluster for this program.

Figure 18 shows how the performance of a partitioning structure varies as a function of its distance from the predicted ideal partitioning structure. This diagram averages the results across all benchmarks and shows that partitions near the predicted ideal give the best average performance. The figure demonstrates that Euclidean distance from the predicted ideal structure is a useful means of discriminating good partitions from poor. Furthermore, as discovered in the experiments, partitions in a cluster that is closed to the ideal partition tend to have similar performance (on average, less than $20 \%$ of difference in runtime). The only exception is MATMUL where performance for partitions in the nearest cluster varies. This is because the static features used by our model do not capture some optimizations performed by the backend compiler (as explained in Section 6.1.1).

7.2.4. Importance of Partitioning Choices on Performance. Section 2 has shown that the best heuristic varies across programs. We now consider the importance of specific partitioning characteristics for each program on the 4-core platform. We have considered a number of characteristics that a partitioning algorithm may wish to consider in making partitioning decisions, for example, communication-computation ratio, average push 


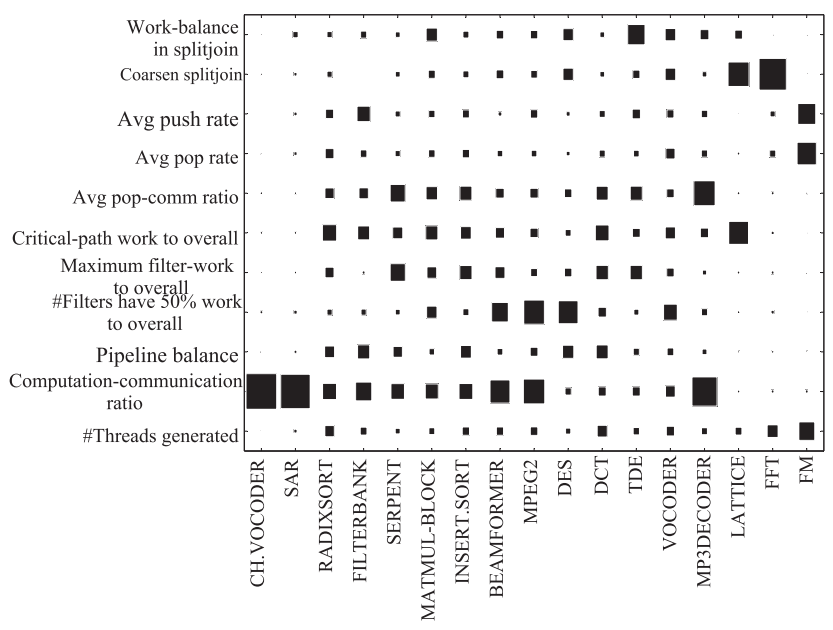

Fig. 19. A Hinton diagram showing the partitioning objectives that are likely to impact performance of each benchmark. The larger the box, the more likely a partitioning objective affects the performance of the respective program.

rate, etc. Figure 19 shows a Hinton diagram illustrating the importance of a number of different partitioning objectives on the performance of each program. Intuitively, this information gives us an indication of those characteristics on which an optimizing heuristic should focus. The larger the box, the more significant the issue for that program. The $x$-axis denotes the programs, the $y$-axis denotes partitioning criteria. Figure 19 shows that each of these objectives has an impact on each program. The computation-to-communication ratio and the critical-path work are important for all programs and extremely important for the CHANNELVOCODER and SAR. For some programs, other features are important. For example, the average pop rate is important for benchmarks MP3Decoder and Serpent. Having a balanced pipeline, however, is less important overall. Some programs are sensitive to all of these objectives, such as RADIXSORT while for some programs, such as FFT, one issue, coarsen the split-join sections, is of overwhelming importance. Using the computation ratio and the criticalpath work only will give a model that has similar performance as the greedy scheme. This diagram illustrates just how hard it is for a heuristic which typically focuses on one or two objectives to find the best partitioning for all programs.

7.2.5. Syntactic Training Benchmarks. Programs within the StreamIt benchmark suite are both small in number and have very different program structures [Thies and Amarasinghe 2010] which means they form a poor training set for cross-validationbased learning. To provide enough training examples, we developed the syntactic benchmark generator. By generating many synthetic benchmarks we can have training programs that are "similar" to the testing programs. More specifically, we were able to generate syntactic benchmarks that are similar to FILTERBANK, FM, and LATTICE. This leads to performance that is closed to the "best found" performance for those testing programs. Syntactic benchmark generation allows us to build a model with sufficient training programs that eventually leads to accurate prediction for the test program.

\subsection{Adapting to a New Platform}

In order to evaluate the portability of our model, we evaluated it on a 8-core Intel platform. Training data was collected from the new platform and used to train the 


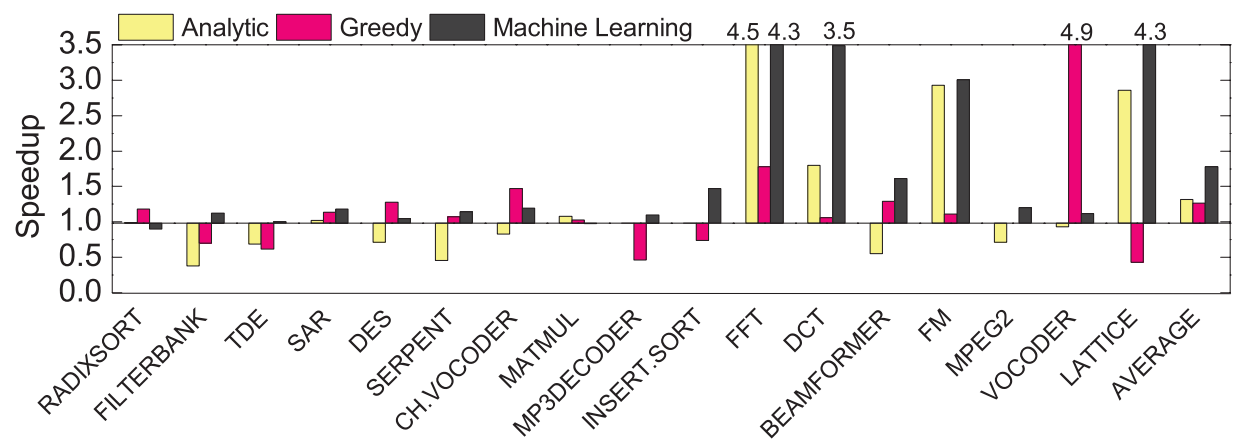

Fig. 20. Performance comparison on the 8-core platform for the analytical model, the greedy partitioner, and the ML-based model.

model. Note that we used the same program features to train the model as used on the 4-core platform. Due to time constraints we had to use relatively fewer partitions for each training program. This will affect the performance of our model.

Figure 20 shows the performance of the different approaches on the 8-core platform. The most striking result is that for some applications, the greedy partitioner does better than it does on the 4-core platform. For DES and FFT, the greedy partitioner achieves $1.28 \times$ and $1.78 \times$ speedup, respectively, compared to the default dynamic programming partitioner when on the 4-core platform, it slowed down these programs to $70 \%$ of the partition generated by the StreamIt default. The greedy partitioner improves the performance of 10 benchmarks on this platform, up to $4.9 \times$ for VOCODER, with an average $1.2 \times$ speedup but gives significant performance slowdowns for 5 programs. The analytical-based model also gives unstable results. It gives an average speedup but only gives noticeable improvement on 4 programs. It, however, gives a significant slowdown on FILTERBANK by more than a factor of 2 .

In contrast to these two approaches, our machine learning approach is more stable across programs, with just one small slowdown in the case of RADIXSORT. On average we achieve $1.8 \times$ improvement across the benchmarks. With the correct amount of training data, this is certain to improve even further. Compared to the analytical model and the greedy heuristic, our model is stable not only across programs but also across platforms. This example demonstrates the portability of our approach.

\section{RELATED WORK}

Stream Graph Partitioning. There is a significant volume of prior work dealing with stream graph partitioning. Dai et al. [2005] proposed a program transformation technique to automatically partitioning packet processing applications for networking processors. Liao et al. [2006] build an affine partitioning framework to map streaming parallelism onto multicore processors. Navarro et al. [2009] construct an analytical model to determine parallelism configurations for pipeline parallelism which contains data parallel pipeline stages. Essentially, these are analytical-based approaches which have to be manually updated when a new platform is targeted. Stream Graph Modulo Scheduling (SGMS) orchestrates execution of StreamIt applications for the IBM Cell processor [Kudlur and Mahlke 2008]. SGMS uses Integer Linear Programming (ILP) formulations to perform partition by aiming to overlap the communication and computation. A similar ILP-solver-based approach has also been used to generate partitions for StreamIt applications targeting on GPUs [Udupa et al. 2009]. Formulating ILP models, however, requires expert knowledge on the underlying architecture. Finding a solution under certain constraints for the ILP formula can be time consuming too. 
Runtime Scheduling for Streaming Parallelism. Gordon et al. exploited coarse-grain task-, data-, and pipeline-level parallelism incorporating with a dynamic task scheduler. Their approach first uses a greedy partitioner to partition a stream graph, then uses a runtime scheduler to execute the partitioned streaming graph onto multicores [Gordon et al. 2002]. FlexStream [Hormati et al. 2009] is a runtime adaptation system that dynamically remaps an already partitioned stream graph according to the number of processors available for heterogeneous multicore systems. Aleen et al. [2010] combine profiling information and execution-time estimation to predict the dynamic behavior of a stream application. Our approach is orthogonal to existing and future scheduling approaches, which significantly reduces the efforts of porting a stream graph partitioner to new architectures or runtime systems.

Machine-Learning-Based Compilation. Machine learning has been successfully applied to optimising both sequential and data parallel programs. Stephenson et al. [2003] have used genetic algorithms to tune the compiler heuristics for sequential programs. Similar approaches also have been used to find either good loop transformations [Pouchet et al. 2008] or compiler flags [Hoste and Eeckhout 2008]. In contrast to these online learning algorithms, our predictor learns from prior knowledge obtained by off line training, hence, it has a much lower overhead in deployment. In our previous work [Wang and O'Boyle 2009; Grewe et al. 2011], we have built machine learning models to map data parallel programs onto multicores. The Qilin compiler [Luk et al. 2009] divides parallel loops between CPUs and GPUs using regression-based prediction. All approaches focus on fixed targets, such as per-loop or a fixed number of tasks. This work is distinct from previous ones in that we target on streaming parallelism which contains both data and pipeline parallelism and the prediction target (e.g., the transformation sequence) is unbounded.

\section{DISCUSSION}

This article presents a novel machine-learning-based approach to partition streaming programs that have complex parallel structures. It is the first time machine learning is shown applicable for compilation problems that target unbounded graph structures.

Our approach was implemented in the StreamIt compiler and evaluated using the StreamIt benchmark suite. While StreamIt is a sophisticated programming language with an open-source compiler framework, it has a number of limitations. One particular problem is the majority of the StreamIt benchmarks are static-rate programs and the current StreamIt compiler only provides basic, unoptimized support for dynamic-rate programs [Thies and Amarasinghe 2010]. Because of this, we did not perform extensive evaluation for dynamic-rated programs in experiments. We expect that compiler-based profiling techniques [Kudlur and Mahlke 2008] are needed for programs whose communication rates and work cannot be statically determined.

Our machine learning approach implicitly models the runtime scheduling polices (along with the rest of the system, e.g., hardware and operating systems) by automatically learning from training data. In StreamIt the partitioned code is mapped down to pthreads which are then scheduled by the Linux kernel to processor cores using its default policy. Rather than experimenting with this OS policy, we simply learn its behavior implicitly when training the model. Should a new scheduling scheme be targeted, the machine learning model may need to be retrained; this cost has to pay for any other heuristic- and analytical-based approaches too. However, our machine-learning-based approach has great advantages when compared to heuristics and analytical models. First, it makes no assumption of the underlying platform and scheduling policies. Instead, it automatically models and observes the behavior of the hardware and runtime by learning from data. Second, since the process of generating data and training a model 
are done automatically, our approach requires far less effort and human involvement when compared to tuning a new heuristic by hand.

\section{CONCLUSIONS}

This article has presented an automatic and portable compiler-based approach to partitioning streaming programs for multicores, providing a significant performance improvement over hardwired heuristics. Using machine learning techniques, our compiler predicts the ideal partition structure of a streaming application, allowing us to quickly search the transformation space without running the code. In addition to the predictive model, we have developed a microkernel streaming program generator which automatically generates small training examples for the predictive model. We demonstrated our approach by mapping StreamIt applications onto two multicore platforms. On average, we achieve a $1.90 \times$ speedup over the StreamIt default scheme on a 4-core platform. Compared to a recently proposed analytical-based model, our approach achieves on average a $1.79 x$ performance improvement. When our approach was ported to an 8-core machine, we were able to achieve a $1.80 \times$ improvement over the StreamIt default. Future work will consider incorporating our compiler framework with runtime task scheduler to dynamically exploit task, data, and pipeline parallelism during program execution time.

\section{REFERENCES}

Aleen, F., Sharif, M., AND Pande, S. 2010. Input-driven dynamic execution prediction of streaming applications. In Proceedings of the $15^{\text {th }}$ ACM-SIGPLAN Symposium on Principles and Practice of Parallel Programming (PPoPP'10). 315-324.

Asanovic, K., Bodik, R., Demmel, J., Keaveny, T., Keutzer, K., Kubiatowicz, J., Morgan, N., Patterson, D., Sen, K.,WAwrzynek, J.,Wessel, D., AND Yelick, K. 2009. A view of the parallel computing landscape. Comm. ACM 52, 56-67.

Bishop, C. M. 2006. Pattern Recognition and Machine Learning. Springer.

Bui, T. N. AND Jones, C. 1992. Finding good approximate vertex and edge partitions is np-hard. Inf. Process. Lett. $42,3$.

Culler, D. E., Karp, R. M., Patterson, D., Sahay, A., Santos, E. E., Et Al. 1996. LogP: A practical model of parallel computation. Comm. ACM 39, 11.

DAI, J., HuAnG, B., Li, L., AND HaRrison, L. 2005. Automatically partitioning packet processing applications for pipelined architectures. In Proceedings of the ACM-SIGPLAN Conference on Programming Language Design and Implementation (PLDI'05). 237-248.

Duda, R. O., Hart, P. E., AND Stork, D. G. 2000. Pattern Classification, $2^{\text {nd }}$ Ed. Wiley-Interscience.

Gordon, M. I., Thies, W., And Amarasinghe, S. 2006. Exploiting coarse-grained task, data, and pipeline parallelism in stream programs. In Proceedings of the $12^{\text {th }}$ International Conference on Architectural Support for Programming Languages and Operating Systems (ASPLOS'06). 151-162.

Gordon, M. I., Thies, W., Karczmarek, M., Lin, J., Meli, A. S., Lamb, A. A., Leger, C., Wong, J., Hoffmann, H., Maze, D., And Amarasinghe, S. 2002. A stream compiler for communication exposed architectures. In Proceedings of the $10^{\text {th }}$ International Conference on Architectural Support for Programming Languages and Operating Systems (ASPLOS'02). 291-303.

Grewe, D.,Wang, Z., ANd O'Boyle, M. F. P. 2011. A workload-aware mapping approach for data-parallel programs. In Proceedings of the $6^{\text {th }}$ International Conference on High Performance and Embedded Architectures and Compilers (HiPEAC'11). 117-126.

Hormati, A. H., Choi, Y., Kudlur, M., Rabbah, R., Mudge, T., and Mahlke, S. 2009. Flextream: Adaptive compilation of streaming applications for heterogeneous architectures. In Proceedings of the $18^{\text {th }}$ International Conference on Parallel Architectures and Compilation Techniques. 214-223.

Hoste, K. And Eeckhout, L. 2008. Cole: Compiler optimization level exploration. In Proceedings of the $6^{\text {th }}$ Annual IEEE / ACM International Symposium on Code Generation and Optimization (CGO'08). 165-174.

Kudlur, M. AND MAHLKe, S. 2008. Orchestrating the execution of stream programs on multicore platforms. In Proceedings of the ACM SIGPLAN Conference on Programming Language Design and Implementation (PLDI'08). 114-124. 
Kwok, Y. AND Ahmad, I. 1999. Static scheduling algorithms for allocating directed task graphs to multiprocessors. ACM Comput. Surv. 31, 4.

Lee, E. A. and Messerschmitt, D. G. 1987. Synchronous data flow. Proc. IEEE 75, 9.

LiAo, S.-W., Du, Z., Wu, G., AND LuEH, G.-Y. 2006. Data and computation transformations for brook streaming applications on multiprocessors. In Proceedings of the International Symposium on Code Generation and Optimization (CGO'06). 196-207.

LuK, C.-K., Hong, S., AND KIM, H. 2009. Qilin: Exploiting parallelism on heterogeneous multiprocessors with adaptive mapping. In Proceedings of the $42^{\text {nd }}$ Annual IEEE/ACM International Symposium on Microarchitecture (MICRO'09). 45-55.

Moss, E., Utgoff, P., Cavazos, J., Brodley, C., Scheeff, D., Precup, D., And Stefanovic, D. 1998. Learning to schedule straight-line code. In Proceedings of the Conference on Advances in Neural Information Processing Systems (NIPS'97). 929-935.

Navarro, A., Asenjo, R., TABik, S., and Cascaval, C. 2009. Analytical modeling of pipeline parallelism. In Proceedings of the $18^{\text {th }}$ International Conference on Parallel Architectures and Compilation Techniques. 281-290.

Pelleg, D. AND Moore, A. W. 2000. X-means: Extending k-means with efficient estimation of the number of clusters. In Proceedings of the $17^{\text {th }}$ International Conference on Machine Learning (ICML'00). 727-734.

Pouchet, L.-N., Bastoul, C., Cohen, A., And Cavazos, J. 2008. Iterative optimization in the polyhedral model: Part ii, multidimensional time. In Proceedings of the ACM-SIGPLAN Conference on Programming Language Design and Implementation (PLDI'08). 90-100.

Ramamritham, K. and Stankovic, J. A. 1984. Dynamic task scheduling in hard real-time distributed systems. IEEE Softw. 1, 3.

SARKAR, V. 1991. Automatic partitioning of a program dependence graph into parallel tasks. IBM J. Res. Devel. 35, 5-6.

Schwarz, G. 1978. Estimating the dimension of a model. Ann. Statist. 6, 2.

Sherwood, T., Perelman, E., Hamerly, G., and Calder, B. 2002. Automatically characterizing large scale program behavior. In Proceedings of the $10^{\text {th }}$ International Conference on Architectural Support for Programming Languages and Operating Systems (ASPLOS'02). 45-57.

Stephens, R. 1997. A survey of stream processing. Acta Informatica 34, 7, 491-541.

Stephenson, M. and Amarasinghe, S. 2005. Predicting unroll factors using supervised classification. In Proceedings of the International Symposium on Code Generation and Optimization (CGO'05). 123-134.

Stephenson, M., Amarasinghe, S., Martin, M., and O'Reilly, U.-M. 2003. Meta optimization: Improving compiler heuristics with machine learning. In Proceedings of the ACM SIGPLAN Conference on Programming Language Design and Implementation (PLDI'03). 77-90.

Thies, B., Karczmarek, M., and Amarasinghe, S. 2001. Streamit: A language for streaming applications. In Proceedings of the International Conference on Compiler Construction (CC'01).

THIES, W. 2009. Language and compiler support for stream programs. Ph.D. thesis. Massachusetts Institute of Technology, Cambridge, MA. http://groups.csail.mit.edu/commit/papers/09/thies-phd-thesis.pdf.

Thies, W. and Amarasinghe, S. 2010. An empirical characterization of stream programs and its implications for language and compiler design. In Proceedings of the $19^{\text {th }}$ International Conference on Parallel Architectures and Compilation Techniques (PACT'10).

Tournavitis, G.,Wang, Z., Franke, B., And O’Boyle, M. F. 2009. Towards a holistic approach to auto parallelization: Integrating profile-driven parallelism detection and machine-learning based mapping. In Proceedings of the ACM-SIGPLAN Conference on Programming Language Design and Implementation (PLDI'09). 177-187.

Udupa, A., Govindarajan, R., and Thazhuthaveetil, M. J. 2009. Software pipelined execution of stream programs on gpus. In Proceedings of the $7^{\text {th }}$ Annual IEEE/ACM International Symposium on Code Generation and Optimization (CGO'09). 200-209.

Wang, Z. AND O’Boyle, M. F. 2009. Mapping parallelism to multi-cores: A machine learning based approach. In Proceedings of the $14^{\text {th }}$ ACM SIGPLAN Symposium on Principles and Practice of Parallel Programming (PPoPP'09). 75-84.

Wang, Z. AND O’Boyle, M. F. 2010. Partitioning streaming parallelism for multi-cores: A machine learning based approach. In Proceedings of the $19^{\text {th }}$ International Conference on Parallel Architectures and Compilation Techniques (PACT'10). 307-318.

Received September 2011; revised September 2012; accepted January 2013 